\title{
A STUDY OF THE METHOD OF RECONSTRUCTING THE BIONIC SCAFFOLD FOR REPAIRING DEFECTIVE BONE BASED ON TISSUE ENGINEERING
}

\author{
Hanqiang Liu, Qingxi Hu, Limin Li, Minglun Fang \\ Rapid Manufacturing Engineering Center, Shanghai University, Shanghai 200444, \\ P.R.China; Email: hugingxi@mail.shu.edu.cn.
}

\begin{abstract}
Bionic modeling technology of bone defect repair plays a key role in the process of repairing defective bone based on tissue engineering. In this paper, via studying the structure of bone defect repair, new concept of bionic scaffold is put forward and a new method of integrating medical image processing, 3D reconstruction and CAD technology to construct the bionic scaffold of repairing bone defect is presented. Using the system implemented by this method, the bionic scaffold of repairing a skull is constructed. The evaluation of porosity of scaffold is also analyzed.
\end{abstract}

Key words: bone defect repair, bionic scaffold, modeling, tissue engineering.

\section{INTRODUCTION}

Repairing bone defect based on tissue engineering is a research hotspot for the moment. It comes down to many aspects such as bionic modeling of repair, materials of repair, processing of repair and tests of osteoblasts seeding etc. Among these, bionic modeling of repair is one of the key technologies.

At present, for bionic modeling of bone repair, researchers mostly adopt conventional CAD methods ${ }^{1-2}$. Woodfield, $T$. in Canterbury University of

This project was supported by Shanghai Education Key Foundation 03AZ01

Please use the following format when citing this chapter:

Liu, Hanqiang, Hu, Qingxi, Li, Limin, Fang, Minglun, 2006, in International Federation for Information Processing (IFIP), Volume 207, Knowledge Enterprise: Intelligent Strategies In Product Design, Manufacturing, and Management, eds. K. Wang, Kovacs G., Wozny M., Fang M., (Boston: Springer), pp. 650-657. 
New Zealand and Manjubala, I. in Max Planck Research Community of Germany both have utilized conventional CAD technology combining processing technology to obtain the porestructure of bone repair ${ }^{3-4}$. Professor LU Bingheng and his team in Xi'An JIAOTONG University of China have obtained bioceramics artificial bone which possesses microstructure of $200 \sim 600 \mu \mathrm{m}$ in aperture size by using CAD software combining rapidprototyping (RP) ${ }^{5}$. MAO Ya in WUHAN University of Technology of China presents a design method of bionic bone possessing similar 3D structures as real bone by utilizing fractal technology ${ }^{6}$.

Constructing model of repair via conventional CAD technology needs to be accomplished by professional technicians holding CAD modeling technology, which is a bottleneck to implement the industrialization of repairing bone defect. Hence, based on the study of medical image processing, 3D reconstruction and CAD modeling technology, a new method is established to obtain the repair bionic scaffold from bone medical tomography images in this paper, and meanwhile a professional system of constructing repair bionic scaffold is developed.

\section{CONCEFT OF THE FEATURES OF REPAIR BIONIC SCAFFOLD}

Repair bionic scaffold is the carrier suitable for histiocytes to grow which must meet the requirements of tissue engineering. According to the requirements of tissue engineering and rapid manufacturing, in this paper, the features of repair bionic scaffold are classified as external model feature (external feature) and internal model features (internal features), and the internal features are classified as macrostructure feature (macrostructure) and microstructure feature (microstructure), Figure 1 shows the classification.

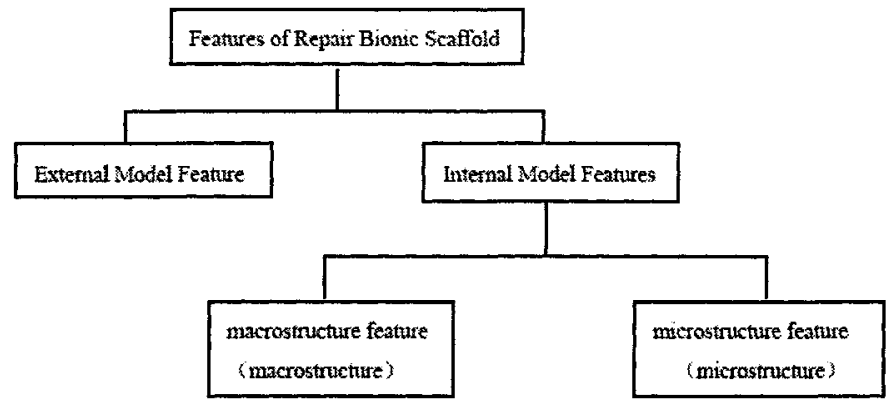

Figure 1. Classification of the features of repair bionic scaffold 
External feature is the external shape feature of repair bionic scaffold, which is the shape of repair. The shape of scaffold should fit the shape of bone defect regions and should have continuous curvatures as possible as the surface of fine bone sections.

Internal features are the internal porestructures of scaffold that offer the environment of osteoblasts growing, and they are the passages through which osteoblasts can get nurture and oxygen. Macrostructure is the porestructure that is constructed in scaffold through modeling technology and it should be constructed according to the requirements of processing. Microstructure is the porestructure formed in scaffold through forming technology, which is regulated by materials and processing. Constructing macrostructure and microstructure is to guarantee that scaffold has favorable porosity and interconnectivity meeting the requirements of tissue engineering.

The modeling methods presented in this paper are used to construct the external feature and macrostructure of scaffold.

\section{METHOD OF CONSTRUCTING THE EXTERNAL MODEL FEATURE OF REPAIR BIONIC SCAFFOLD}

3D reconstruction from tomography images is one of the key steps in the process of constructing repair bionic scaffold from bone tomography images. At the present time, there are mainly three ways of $3 \mathrm{D}$ reconstruction from images:(1) Generating fitting surface through contours of every layer of image (surface reconstruction based on contours). (2) Generating isosurface directly from $3 \mathrm{D}$ volume data (surface reconstruction based on volume data) (3) Enduing color and opacity for every voxel data to render model directly, not to constructing surface ${ }^{7}$. Because scaffold needs to be processed in solid, and generally bones have complex features and the requirements of interaction and calculation amount must be counted, the method of surface reconstruction on volume data is adopted in this paper to construct the model of whole bone, external feature and macrostructure of scaffold.

By the method of surface reconstruction based on volume data, a serial of CT images of a skull with defect from Shanghai X People's Hospital have been reconstructed into 3D model, as shown in Figure 2: 

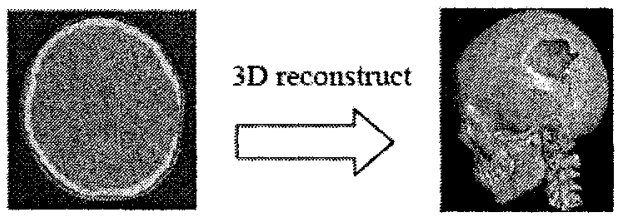

Figure 2.3D reconstruction of a whole skull

Here, a method of constructing the external feature of repair bionic scaffold is presented as followed: firstly, by programming, pixels are filled up in defect regions of bone images; secondly, Boolean operation is proceeded between the pixels of filling up sections and the pixels of original bone images; finally all filling up sections are 3-dimensionally (3D) reconstructed to get the favorable external feature of scaffold.

Figure 3 shows this process of constructing the external feature of the skull repair bionic scaffold through the method above.
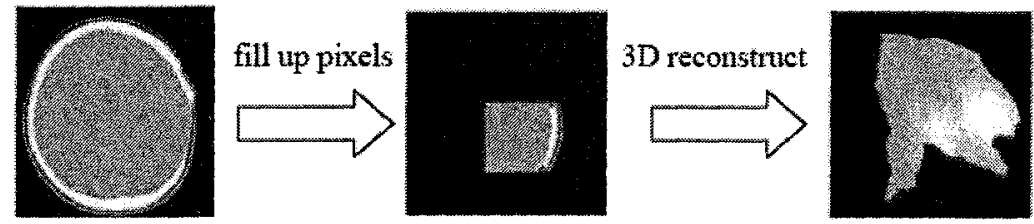

Figure 3. Process of constructing the external feature of repair bionic scaffold

\section{CONSTRUCTING THE MACROSTRUCTURE OF REPAIR BIONIC SCAFFOLD}

\subsection{Requirements of constructing macrostructure}

In the view of tissue engineering, the internal features of repair bionic scaffold should possess appropriate porosity to plant ample osteoblasts in it, appropriate aperture to facilitate attachment and growth of osteoblasts. On the other hand, the structures of internal features should have as high interconnectivity as possible to guarantee that osteoblasts can obtain as ample nurture and oxygen supply as possible. Hence, the requirements of constructing macrostructure are that it had best be fully interconnected, its 
apertures should range from $200 \mu \mathrm{m}$ to $400 \mu \mathrm{m}$ and the whole porosity of repair bionic scaffold should exceed $75 \%{ }^{8}$.

\subsection{Method of constructing macrostructure}

At the present time, there are mainly two methods of constructing internal porestruture of repair bionic scaffold as followed: one is to construct porestruture through the porosity of bionic material (namely microstructure in this paper), this method emphasizes on processing technology to control pores, which has been applied successfully to tissue engineering of cartilage, the most obvious shortcoming of this method is that it is difficult to control porosity; and it is difficult to implement the industrialization of repair bionic scaffold; another method is to construct macrostructure through conventional CAD modeling technology, favorable porosity can be obtained by this method, but, this method is difficult to be implemented.

Here a new method as followed is put forward to construct the macrostructure of repair bionic scaffold:

(1) Choosing the negative of macrostructure (spatial scaffold structure)

(2) Utilizing programming to obtain the crossing sections from intersecting between the negative of macrostructure and the plane that every bone tomography image exists in.

(3) Proceeding Boolean operation between the pixels in filling up sections of every bone image (seen as above) and corresponding cross sections of the negative of macrostructure derived from the step (2).

(4) 3D reconstructing all filling up sections after processed in the step (3) to obtain the macrostructure of scaffold.

There are two key points with this method: one is the calculation of cross sections of the negative of macrostructure; another is to proceed Boolean operation between the pixels in the filling up sections and corresponding cross sections of the negative of macrostructure. The negative of macrostructure is a kind of spatial structure. The law that the negative of macrostructure varies along the increasing axis (generally $z$ axis) of all layers of images is studied, and then the crossing sections at corresponding position of every image are calculated. This process is implemented though programming. The process of Boolean operation between filling up sections and cross sections of negative of macrostructure is within pixels which sets the gray scale values of pixels in cross sections out of the ranges of the gray scale value of bone pixels, thus macrostructure in the scaffold can be formed when all filling up sections are 3D reconstructed.

There are mainly two methods in constructing the shape of macrostructure: One is that the repair bionic scaffold with constructing macrostructure in it is has regular structure, by this method larger porosity 
$(>80 \%)$ can be obtained, for example cylinder can be chosen as the shape of solid not as the shape of pore, and the scaffold with macrostructure constructed in it by this method has lower mechanical strength, and it is more suitable to repair cartilaginous tissue; another method is to choose regular structure as the shape of pore. The scaffold with macrostructure constructed through the second method has higher mechanical strength but lower porosity than the scaffold by the first method, and it is more applicable to repairing bones. According to practical requirements, the regular shape can be cylinder, square column, prism and etc. the intersecting angles between columns can be right angle and bevel. A zooming-in view of the negative of macrostructure adopting intersecting cylinder in spatial rightangle is shown in Figure 4:

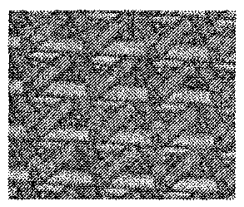

Figure 4. A zooming-in view of the negative of macrostructure

The negative of macrostructure obtained by programming is a spatial structure that is derived from intersecting between many columns threedimensionally. The negative of macrostructure is fully interconnected (as shown in Figure 4 and Figure 5), so the macrostructure of repair bionic scaffold is also fully interconnected.

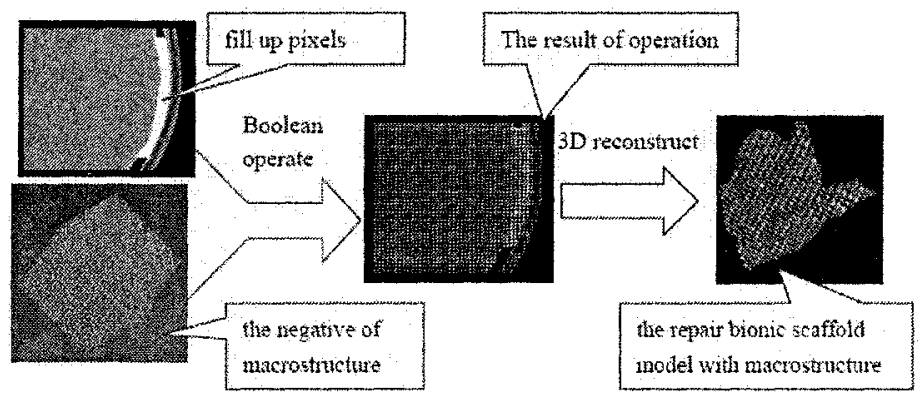

Figure 5. Process of constructing microstructure of repair bionic scaffold

Repair bionic scaffold is a kind of individual model whose external shape and macrostructure both have complex features, and it is difficult to be manufactured in solid by conventional processing methods. RP has excellent 
processing flexibility, which can manufacture models with complex features in high precision; so, the 3D model of scaffold is saved as STL format applicable to RP methods.

Through setting some parameters of macrostructure such as aperture and interval of pores and adjusting the porosity of scaffold, the macrostructure of repair bionic scaffold can be obtained. Favorable porosity and interconnectivity of repair bionic scaffold can be obtained through constructing macrostructure via this method and constructing microstructure via processing. The process of constructing repair bionic scaffold through this method is shown in Figure 5:

\subsection{Evaluation of porosity of repair bionic scaffold}

The porosity of repair bionic scaffold includes two kinds: the porosity of macrostructure derived from modeling and that of microstructure formed through processing. Here, $P$ represents the whole porosity, $P_{1}$ the porosity of macrostructure, $\mathrm{P}_{2}$ the porosity of microstructure, then, $\mathrm{P}$ can be obtained by Eq. (1):

$$
P=P_{1}+P_{2}
$$

$\mathrm{P}_{1}$ can be derived through measuring the volumes of repair bionic scaffold. The volume $V_{1}$ of repair bionic scaffold without macrostructure and the volume $V_{2}$ of repair bionic scaffold with macrostructure are respectively measured, then, $P_{1}$ can be obtained by Eq. (2):

$$
P_{1}=\left(1-V_{2} / V_{1}\right) \times 100 \%
$$

Here, RP processing is adopted to obtain the solid of repair bionic scaffold; the material of repair bionic scaffold is mixed powder of $\beta$ tricalcium phosphate ( $\beta$-TCP) and adhesive (mixture of epoxy resin and nylon). Porestructure (microstructure) can be formed when scaffold solid is burned to hard ceramic body under high temperature because of volatilization of the adhesive. Supposed that the volume ratio of $\beta$-TCP and adhesive is $1: \beta$, the porosity $\mathrm{P}_{2}$ can be derived by Eq. (3):

$$
P_{2}=V_{2} / V_{1} \times(\beta \div(1+\beta)) \times 100 \%
$$

Synthesizing Eq. (1), Eq. (2) and Eq. (3) to obtain the whole porosity of repair bionic scaffold as shown in Eq. (4): 


$$
P=\left(\left(1-V_{2} / V_{1}\right)+V_{2} / V_{1} \times(\beta \div(1+\beta))\right) \times 100 \%
$$

The methods mentioned above is adopted to construct the external feature and macrostructure of the repair bionic scaffold of the skull above, the shape of the pores of macrostructure is cylinder, the intersecting angle is right-angle the aperture of pore is $320 \mu \mathrm{m}$, the interval of pores is $400 \mu \mathrm{m}$, and the volume ratio of $\beta$-TCP and adhesive is $1: 1$, finally the whole porosity $\mathrm{P}=80.57 \%$ is obtained by Eq. (4). As can be seen, repair bionic scaffold with favorable porosity to plant osteoblasts can be obtained by using the methods presented in this paper combining processing technology.

\section{CONCLUSION}

This paper presents the concept and classifications of the features of repair bionic scaffold. The calculation method of porosity and the modeling method of scaffold for repairing bone defect are established. A professional system for repairing bone defect is also developed. It will promote the development of biomanufacturing technology.

\section{REFERENCES}

1. SHI Zhen-sheng, HU Qing-xi, WU Mao-liang, DAI Chun-xiang, FANG Ming-lun, (2004), Modeling Technology of Artificial Bone Substitute Based on Reverse Engineering Journal OF Shanghai University (English Edition), vol. 8, pp. 28-30.

2. LI Limin, GUO Guifang, HU Qingxi, WU Maoling, (2005), Fabircation of 3D Porous Scaffolds for the Bone Tsissue Engineering Manufacturing Information Engineering of China5, vol. 34, pp. 86-88.

3. Woodfield T, et al. (2005), Polymer scaffolds fabricated with pore-size gradients as a model for studying the zonal organization within tissue-engineered cartilage constructs Tissue Engineering vol. 11, pp. 1297-1311.

4. Manjubala I, et al. (2005), Biomimetic mineral-organic composite scaffolds with controlled internal architecture, Journal of Materials Science: Materials in Medicine, vol. 16, pp. $1111-1119$

5. Li Xiang, Li Dichen, Su Yanping, Lu Bingheng, (2005), Structure design and fabrication of $\beta$-tricalcium phosphate artificial bone based on rapid prototyping technique, Journal of Xi'an Jiaotong University, vol. 39, pp. 9-12.

6. Mao Ya, Cao Zuobing, Yu Xinming, Rao Gang, (2004), 3D Bionics Artif icial Bone Design Based on Medical Images, Chinese Journal of Stereologyand Image Analysis, vol. 9 pp. 160-163.

7. Tang Guo, Zhao Xiaodong, Wang Yuanmei, (1998), Three-dimension medical image segmentation and visualization, Chinese Computers, vol. 20, pp. 204-209.

8. Laurence Marcelle Mathieua, et al. (2006), Architecture and properties of anisotropic polymer composite scaffolds for bone tissue engineering, Biomaterials, vol. 27, pp. 905916. 